\title{
DEFICIÊNCIA DE FERRO NO ESTIRÃO PUBERTÁRIO
}

\section{IRON DEFICIENCY DURING PUBERTAL GROWTH SPURT}

\author{
Carmen Lúcia de Almeida Santos ${ }^{1,2}$, Marco Akerman ${ }^{3}$, Odival Faccenda $^{4}$, \\ Lourdes Conceição Martins ${ }^{5}$, Lígia de Fátima Nóbrega Reato ${ }^{6}$
}

\begin{abstract}
RESUMO:
Introdução: a deficiência de ferro representa um agravo à saúde, estando associada a prejuízos na capacidade produtiva dos indivíduos, no desenvolvimento cognitivo e na imunocompetência. Objetivo: verificar a prevalência de deficiência de ferro em adolescentes no período do estirão pubertário. Método: realizou-se estudo transversal, com dados clínicos, laboratoriais e socioeconômicos de adolescentes matriculados no Serviço de Hebiatria da Faculdade de Medicina do ABC, na cidade de Santo André, SP. Foram coletados dados de 255 prontuários que continham história clínica completa, incluindo exame físico, classificação do desenvolvimento puberal (Tanner), peso, estatura, inquérito alimentar recordatório e os seguintes exames laboratoriais: hemograma, ferro sérico, saturação transferrina e parasitológico de fezes. Resultados: Dos 255 prontuários estudados, $162(63,5 \%)$ eram do grupo de jovens que estavam no estirão pubertário e $93(36,5 \%)$ pertenciam ao grupo dos que estavam fora do estirão. A presença da deficiência de ferro foi maior entre os adolescentes mais jovens. Houve frequência de deficiência de ferro em $37(14,5 \%)$, sendo $24(16 \%)$ em adolescentes no estirão e $13(11,5 \%)$ nos fora do estirão. Em relação ao sexo, constatou-se que dos 37 adolescentes que apresentaram deficiência de ferro, $24(64,46 \%)$ pertenciam ao sexo masculino e $13(35,14 \%)$ ao feminino. Conclusão: a deficiência de ferro ocorreu com maior frequência nos adolescentes do sexo masculino durante o estirão pubertário e naqueles que praticavam esportes.
\end{abstract}

Palavras-chave: adolescência; estirão e deficiência ferro.

\section{ABSTRACT:}

Introduction: iron deficiency represents a serious injury to the health and it is associated with damages to the productive capacity of individuals, cognitive development and immune competence. Objective: verify the prevalence of iron deficiency in adolescents during the pubertal growth spurt. Methods: it was made a cross sectional study with clinical, laboratory and socioeconomic adolescents enrolled in Hebiatry Service of the Faculty of Medicine of ABC, in the city of Santo André, SP. Data were collected from 255 medical records containing complete medical history, including physical examination, classification of pubertal development (Tanner), weight, height, diet recall survey and the following laboratory tests: blood count, serum iron, transferrin saturation and stool tests. Results: of the 255 records studied, 162 $(63.5 \%)$ were in the group of young people who were in the pubertal growth spurt and $93(36.5 \%)$ belonged to the group who were outside the spurt. The presence of iron deficiency was higher among younger adolescents. There frequency of iron deficiency in $37(14.5 \%)$, and $24(16 \%)$ adolescents in the spurt and $13(11.5 \%)$ out of the stretch. Regarding gender, it was found that of the 37 adolescents who had iron deficiency, $24(64.46 \%)$ were males and $13(35.14 \%)$ were females. Conclusion: iron deficiency occurred more frequently in male adolescents during pubertal growth spurt and those who practiced sports.

Key words: adolescence; growth spurt; iron deficiency.

\section{INTRODUÇÃO}

A Organização Mundial de Saúde define adolescência como o período do desenvolvimento humano caracterizado pela transição entre a infância e a vida adulta, com mudanças somáticas, psicológicas e sociais. Compreende a puberdade, quando se observa o crescimento físico acelerado, com a ocorrência do pico de crescimento estatural (estirão) e da maturação biológica (óssea e sexual) ${ }^{1}$.
A puberdade manifesta-se através do aparecimento dos caracteres sexuais secundários do indivíduo. Mudanças observáveis externamente são o aparecimento de pêlos pubianos em ambos os sexos, dos seios nas meninas e do aumento do tamanho do pênis e testículos nos meninos. Internamente as mudanças ficam por conta do aumento gradual dos hormônios produzidos pelo ovário nas meninas, e pelas células testiculares nos meninos ${ }^{2}$.

Dados do National Health and Nutrition Examination Survey (NHANES- III) descritos por

\footnotetext{
Faculdade de Ciências Médicas da Universidade Federal da Grande Dourados - MS.

2 Disciplina de Pediatria. Faculdade de Ciências Médicas da Universidade Federal da Grande Dourados - MS.

3 Disciplina de Saúde Coletiva da Faculdade de Medicina do ABC, Santo André/SP.

4 Professor Titular. Universidade Estadual de Mato Grosso do Sul.

5 Professora do Programa de Pós-Graduação em Saúde Coletiva da Universidade Católica de Santos - UNISANTOS.

6 Disciplina de Hebiatria. Departamento de Pediatria. Faculdade de Medicina do ABC, Santo André/SP.

Corresponding author: carmen.elias@globo.com
}

Suggested citation: Santos CLA, et al. Iron deficiency during pubertal growth spurt. Iron deficiency during pubertal growth spurt. J. Hum. Growth Dev. 2012; 22(3): 341-347

Manuscript submitted Jul 16 2011, accepted for publication Aug 302012. 
Frutuoso, Vigantzky e Gambardella ${ }^{3}$ referem que o efeito do estirão de crescimento, que ocorre durante a adolescência, repercute no metabolismo e na necessidade de ferro. Os adolescentes apresentam de uma maior demanda desse nutriente em decorrência da expansão do volume sanguíneo total e ao pool de ferro, consequência do aumento da quantidade de massa magra. Para as meninas, acrescenta-se, ainda, a perda deste mineral ocorrida na menstruação. Desta forma, a necessidade de ferro praticamente dobra durante a puberdade.

Para vários autores, dentre eles Fagioli e Nasser ${ }^{4}$ e Lima et al. ${ }^{5}$, durante a adolescência as necessidades nutricionais têm maior relação com a idade fisiológica do que com a cronológica, estando diretamente ligada de forma proporcional à velocidade de crescimento e às mudanças da composição corporal. Para o corpo humano crescer e se manter realiza inúmeras reações químicas, que são determinadas pelo metabolismo. É papel dos nutrientes, entre eles o ferro, ingeridos através da alimentação, fornecer energia e participar do processo metabólico que origina a manutenção e crescimento dos organismos.

A anemia ferropriva é considerada um dos principais problemas de saúde pública em todo o mundo. A deficiência de ferro alimentar, principal responsável pela desnutrição, mesmo na forma moderada, representa um agravo à saúde, estando associada a prejuízos na capacidade produtiva dos indivíduos, no desenvolvimento cognitivo e na imunocompetência6.

Desta maneira, o objetivo é avaliar a relação entre o estirão de crescimento a prevalência de deficiência de ferro na adolescência.

\section{MÉTODO}

Trata-se de estudo retrospectivo, descritivo analítico, transversal, realizado no período de juIho a dezembro de 2004, em que foram analisados dados de prontuários de 255 adolescentes entre 10 a 20 anos incompletos, matriculados no Ambulatório de Adolescentes do Centro de Saúde Escola da Faculdade de Medicina do ABC, distribuídos em dois grupos: masculino e feminino. Foram excluídos os prontuários dos adolescentes que apresentavam patologias sistêmicas ou portadores de parasitoses que espoliam sangue.

O campo de pesquisa é um serviço de atenção primária de saúde, localizado na periferia de Santo André, município da região da Grande ABC Paulista, que adota a seguinte norma: as consultas são agendadas e, por ocasião da matrícula, após avaliação médica, são solicitados exames laboratoriais de rotina: hemograma, ferro sérico (FS), transferrina (CTLF), saturação da transferrina (FS/ CTLF)X100) e protoparasitológico de fezes.

A amostra foi subdividida em dois grupos: adolescentes que estavam no estirão e os que não estavam e que serviram como grupo controle. Considerou-se estirão de crescimento para os meninos os que se encontravam entre os estágios G3 e G4 (desenvolvimento genital) e para as meninas os estágios M2 e M3 (desenvolvimento das mamas), segundo os critérios de Tanner. Para avaliação nutricional, foi calculado o IMC (Índice de Massa Corporal), cujo valor foi comparado como os referenciais do NCHS para cada adolescente.

Os resultados obtidos foram comparados com padrões pré-estabelecidos. Hemoglobina $(\mathrm{Hb})<11,5 \mathrm{~g} / \mathrm{dL}$ e hematócrito $(\mathrm{Ht})<35 \%$ para meninas e rapazes nos estágios iniciais de Tanner.

No período de estirão pubertário adotou-se, como valores críticos, os propostos pela Organização Mundial de Saúde (OMS) considerando-se Hb $<12,0 \mathrm{~g} / \mathrm{dL}$ e $\mathrm{Ht}<35 \%$ para meninas e, para meninos, $\mathrm{Hb}<12,5 \mathrm{~g} / \mathrm{dL}$ e $\mathrm{Ht}<35 \%$; ferro sérico < $40 \mathrm{mcg} / \mathrm{dL}, \mathrm{TIBC}>350 \mathrm{mcg} / \mathrm{dL}$. Para saturação de transferrina foram utilizados $<16 \%$ e $20 \%$ respectivamente para mulheres e homens ${ }^{7}$.

Foram consideradas como variáveis de exposição: idade, sexo, menarca, classificação nutricional, prática de esportes, desenvolvimento puberal, idade da menarca, sintomas e sinais de anemia, tipo de moradia e renda familiar. Como variável resposta foi considerada: a deficiência de ferro, usando a saturação de transferrina

Para verificar a associação entre duas variáveis categóricas utilizou-se o teste qui-quadrado $\left(\chi^{2}\right)$ de Pearson ou o teste exato de Fischer nos casos em que ocorreu uma frequência esperada menor que dois, ou quando mais de $20 \%$ das frequências esperadas foram menores que cinco.

Nas análises que envolveram a variável resposta contínua (porcentagem de saturação de transferrina) utilizou-se o teste t-Student para comparar diferenças entre duas médias. Quando havia mais de duas médias envolvidas usou-se a análise de variância para verificar diferenças significativas, e o teste de Tukey para comparações múltiplas de médias duas a duas. Optou-se por testes paramétricos, pois, no caso da variável saturação de transferrina, as pressuposições de normalidade (teste Kolmogorov-Smirnov) e igualdade entre variâncias (teste de Levene) foram atendidas.

Para verificar se a associação entre as variáveis estudadas são próprias delas mesmas ou interferência de terceiras procedeu-se a uma abordagem multivariada com aplicação da regressão logística. Todos os resultados foram analisados considerando o valor $\mathrm{p}<0,05$ como diferença significativa.

O trabalho foi aprovado pelo Comitê de Ética em Pesquisa da Faculdade de Medicina do ABC (No 003/2006).

\section{RESULTADOS}

Dos 255 prontuários estudados, 162 (63,5\%) eram do grupo de jovens que estavam no estirão pubertário e $93(36,5 \%)$ pertenciam ao grupo dos que estavam fora do estirão. 
Table 1: Prevalência da deficiência de ferro entre os adolescentes de acordo com seu sexo, idade e fase de desenvolvimento. CSEFMABC, 2004

\begin{tabular}{|c|c|c|c|c|c|c|c|c|c|c|c|c|c|}
\hline \multirow{5}{*}{ Idade } & \multirow{5}{*}{ Deficiência de ferro } & \multicolumn{12}{|c|}{ Adolescentes $(n=255)$} \\
\hline & & \multirow{2}{*}{\multicolumn{4}{|c|}{$\begin{array}{l}\text { No estirão }(n=162) \\
\text { Sexo }\end{array}$}} & \multirow{2}{*}{\multicolumn{4}{|c|}{$\begin{array}{l}\text { Fora do estirão }(n=93) \\
\text { Sexo }\end{array}$}} & \multirow{2}{*}{\multicolumn{4}{|c|}{$\begin{array}{l}\text { Total } \\
\text { Sexo }\end{array}$}} \\
\hline & & & & & & & & & & & & & \\
\hline & & \multicolumn{2}{|c|}{ Masculino } & \multicolumn{2}{|c|}{ Feminino } & \multicolumn{2}{|c|}{ Masculino } & \multicolumn{2}{|c|}{ Feminino } & \multicolumn{2}{|c|}{ Masculino } & \multicolumn{2}{|c|}{ Feminino } \\
\hline & & $\mathrm{N}$ & $\%$ & $\mathrm{n}$ & $\%$ & $\mathrm{n}$ & $\%$ & $\mathrm{n}$ & $\%$ & $\mathbf{n}$ & $\%$ & & $\%$ \\
\hline \multirow[t]{2}{*}{$10-$ I 13} & Presente & 5 & $29,4 *$ & 7 & 77,8 & 5 & 71,4 & 3 & 75,0 & 10 & 41,7 & 10 & 76,9 \\
\hline & Ausente & 14 & 23,0 & 51 & 68,0 & 29 & 65,9 & 5 & 13,2 & 43 & 41,0 & 56 & 49,6 \\
\hline \multirow[t]{2}{*}{$13-$ I 16} & Presente & 9 & 52,9 & 2 & 22,2 & 2 & 28,6 & 1 & 25,0 & 11 & 45,8 & 3 & 23,1 \\
\hline & Ausen & 41 & 67,2 & 24 & 32,0 & 9 & 20,5 & 13 & 34,2 & 50 & 47,6 & 37 & 32,7 \\
\hline \multirow[t]{2}{*}{16 - I 20} & Presente & 3 & 17,6 & 0 & 0,0 & 0 & 0,0 & 0 & 0,0 & 3 & 12,5 & 0 & 0,0 \\
\hline & Ausente & 6 & 9,8 & 0 & 0,0 & 6 & 13,6 & 20 & 52,6 & 12 & 11,4 & 20 & 17,7 \\
\hline \multirow[t]{2}{*}{ Total } & Presente & 17 & 100 & 9 & 100 & 7 & 100 & 4 & 100 & 24 & 100 & 13 & 100 \\
\hline & Ausente & 61 & 100 & 75 & 100 & 44 & 100 & 38 & 100 & 105 & 100 & 113 & 100 \\
\hline
\end{tabular}

* A porcentagem foi calculada em relação ao total da coluna $5 / 17=29,4 \%, 14 / 61=23,0 \%$

Quanto à distribuição por idade (Tabela 1) verificou-se que meninas na faixa dos 10 a 13 anos constituíram o grupo em maior proporção da amostra: $119,46,7 \%$ do total, seguidas pelas 101 de 13 a 16 anos e pelas 35 mais velhas (13,7\%).

Como se vê na Tabela 1, a presença da deficiência de ferro foi maior entre os adolescentes mais jovens diminuindo à medida entre os mais velhos. $\mathrm{Na}$ primeira faixa de idade encontrou-se 20 ferro deficientes $(16,8 \%)$, diminuindo para $14(13,9 \%)$ na segunda e para $3(8,6 \%)$ na terceira, porém estas diferenças não foram estatisticamente significativas $\left(\chi_{(2)}^{2}=1,535, p=0,464\right)$.

Quanto à frequência de deficiência de ferro na amostra total constatou-se um percentual de $37(14,5 \%)$, sendo $24(16 \%)$ em adolescentes no estirão e $13(11,5 \%)$ nos fora do estirão. A diferença, portanto, entre os portadores de deficiência de ferro dos adolescentes no estirão e fora dele foi de $4,5 \%$. Esta diferença não foi estatisticamente significativa $\left(\chi^{2}{ }_{(1)}=0,849 ; p=0,357\right)$, ou seja, os indivíduos pertencentes às duas amostras não diferiram em relação a deficiência de ferro.

Em relação ao sexo, constatou-se que dos 37 adolescentes que apresentaram deficiência de ferro, $24(64,46 \%)$ pertenciam ao sexo masculino e 13 $(35,14 \%)$ ao feminino. Esta diferença não foi estatisticamente significativa $\left(\chi_{(1)}^{2}=3,529 ; p=0,060\right)$.
Considerando-se apenas os adolescentes no grupo do estirão esta diferença aumentou: dos 26 que apresentavam deficiência de ferro, 17 (65,4\%) pertenciam ao sexo masculino. Esta diferença atinge uma situação limítrofe $\quad\left(\chi^{2}{ }_{(1)}=3,686\right.$; $p=0,055)$, mas insuficiente para rejeitar a hipótese de que a deficiência de ferro é igual entre os gêneros.

Dos 91 adolescentes que praticavam esporte, $16(17,6 \%)$ possuíam deficiência de ferro e dos 134 que não praticavam $15(10 \%)$ apresentaram deficiência. Esta diferença, ainda que aparentemente alta, também não apresentou diferença significativa $\left(\chi^{2}{ }_{(1)}\right.$ $=2,837 ; p=0,092)$, no entanto observou-se uma tendência de deficiência de ferro no estirão pubertário para os que praticavam esportes.

Conforme dados apresentados na Tabela 2 constatou-se que do total dos adolescentes estudados, $197(77,2 \%)$ eram eutróficos, $18(7,1 \%)$ obesos, 25 (9,8\%) magros e 15 (5,9\%) apresentava sobrepeso. Fazendo esta análise para cada grupo no estirão e fora do estirão separadamente observou-se que dos 127 adolescentes eutróficos que estavam no estirão $19(14,96 \%)$ apresentaram diagnóstico de deficiência de ferro. Dos 70 adolescentes eutróficos que estavam fora do estirão, 6 $(8,57 \%)$ apresentaram diagnóstico de deficiência de ferro.

Table 2: Frequência de deficiência de ferro $X$ Classificação nutricional

\begin{tabular}{|c|c|c|c|c|c|c|c|c|c|c|c|c|c|}
\hline & & \multicolumn{8}{|c|}{ Adolescentes } & \multicolumn{4}{|c|}{ Total } \\
\hline & & \multicolumn{4}{|c|}{$\begin{array}{c}\text { No estirão } \\
\text { Deficiência de ferro }\end{array}$} & \multicolumn{4}{|c|}{$\begin{array}{l}\text { Fora do estirão } \\
\text { Deficiência de ferro }\end{array}$} & & & & \\
\hline & & \multicolumn{2}{|c|}{ Presente } & \multicolumn{2}{|c|}{ Ausente } & \multicolumn{2}{|c|}{ Presente } & \multicolumn{2}{|c|}{ Ausente } & \multicolumn{2}{|c|}{ No estirão } & \multicolumn{2}{|c|}{ Fora do estirão } \\
\hline & & $\mathrm{n}$ & $\%$ & $\mathrm{~N}$ & $\%$ & $n$ & $\%$ & $n$ & $\%$ & $\mathrm{~N}$ & $\%$ & $n$ & $\%$ \\
\hline Clasificação & Eutrofia & 19 & 73,1 & 108 & 79,4 & 6 & 54,5 & 64 & 78,0 & 127 & 78,4 & 70 & 75,3 \\
\hline \multirow[t]{3}{*}{ Nutricional } & Obesidade & 1 & 3,8 & 13 & 9,6 & 2 & 18,2 & 2 & 2,4 & 14 & 8,6 & 4 & 4,3 \\
\hline & Magreza & 5 & 19,2 & 13 & 9,6 & 1 & 9,1 & 6 & 7,3 & 18 & 11,1 & 7 & 7,5 \\
\hline & Sobrepeso & 1 & 3,8 & 2 & 1,5 & 2 & 18,2 & 10 & 12,2 & 3 & 1,9 & 12 & 12,9 \\
\hline Total & & 26 & 100 & 136 & 100 & 11 & 100 & 82 & 100 & 162 & 100 & 93 & 100 \\
\hline
\end{tabular}

Dos 129 adolescentes masculinos, 78 (60,5\%) estavam no estirão pubertário e 51 (39,5\%) fora dele. Entre os adolescentes masculinos que se encontravam no estirão pubertário $17(21,8 \%)$ tinham deficiência de ferro. $O$ estágio G3 foi o que apresentou maior percentual de deficiência de ferro, $11(23,39 \%)\left(\chi^{2}{ }_{(1)}=1,330 ; p=0,249\right)$. No entanto, nos demais estágios esta diferença diminuiu e, consequentemente, não foi encontrada diferença significativa $(p>0,05)$ (Tabela 3$)$. 
Tabela 3: Frequência do diagnóstico de deficiência de ferro em adolescentes do sexo masculino segundo grupos definidos pelo critério de Tanner

\begin{tabular}{cccccc} 
Estágio Tanner Feminino & \multicolumn{2}{c}{ Deficiência de ferro Presente } & Total & $\%$ de diagnóstico \\
& & & $\mathrm{N}$ & $\mathbf{9}$ & 11,1 \\
& $\mathrm{G} 2$ & $\mathrm{G} 1$ & 1 & 15,2 & \\
& $\mathrm{G} 3$ & 11 & $\mathbf{3 3}$ & $\mathbf{4 6}$ & 18,8 \\
& $\mathrm{G} 4$ & 6 & $\mathbf{3 2}$ & 11,1 \\
Total & $\mathrm{G} 5$ & 1 & $\mathbf{9}$ & $\mathbf{1 8 , 6}$
\end{tabular}

Nas meninas, $9(10,7 \%)$ tinham diagnóstico de deficiência de ferro e encontravam-se no estirão, foram identificadas $4(9,5 \%)$ que não se encontravam no estirão. O estágio M4 foi o que apresentou maior percentual de deficiência de ferro, $(16,7 \%)$, contra $(9,7 \%)$ dos que não pertenciam a este estágio (Tabela 4).

Para os demais estágios do critério de Tanner nas adolescentes femininas esta diferença diminuiu houve estatisticamente significativa $(p>0,05)$.

Tabela 4: Frequência do diagnóstico de deficiência de ferro no sexo feminino segundo grupos definidos pelo critério de Tanner

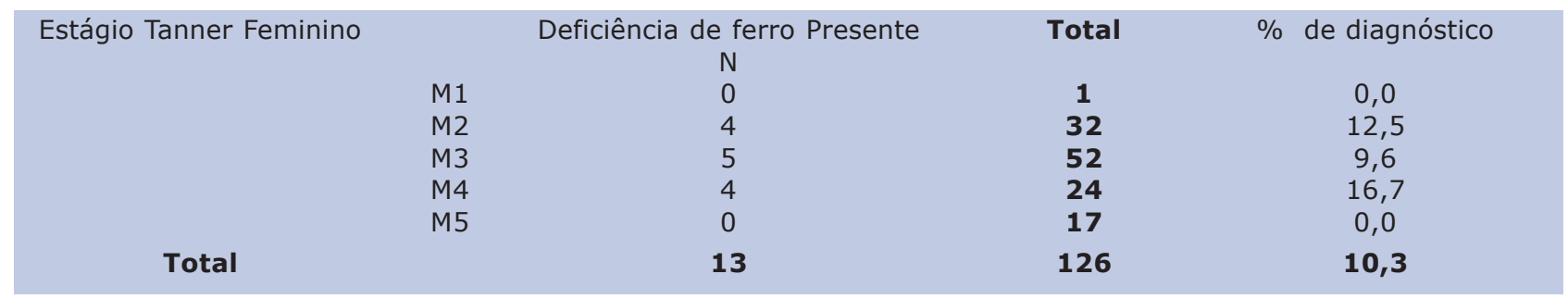

O número das adolescentes na amostra com menarca foi de 66. Do total de adolescentes com menarca, $6(9,1 \%)$ foram identificadas com diagnóstico de deficiência de ferro e nas que não haviam apresentado menarca, $6(10,91 \%)$ apresentavam deficiência de ferro. Esta diferença não foi significativa, $\left(\chi_{(1)}^{2}=0,223 ; p=0,636\right)$, isto é, a deficiência de ferro na amostra de adolescentes do sexo feminino não dependeu do fato da mesma ter tido menarca ou não. Este fato está ilustrado na Figura 1 utilizando-se o valor de saturação de transferrina, onde o percentual médio de saturação de transferrina não diferiu significativamente $\left(t_{(114)}=0,595 ; p=0,553\right)$ entre menarcas e não menarcas, pelo teste t de Student.

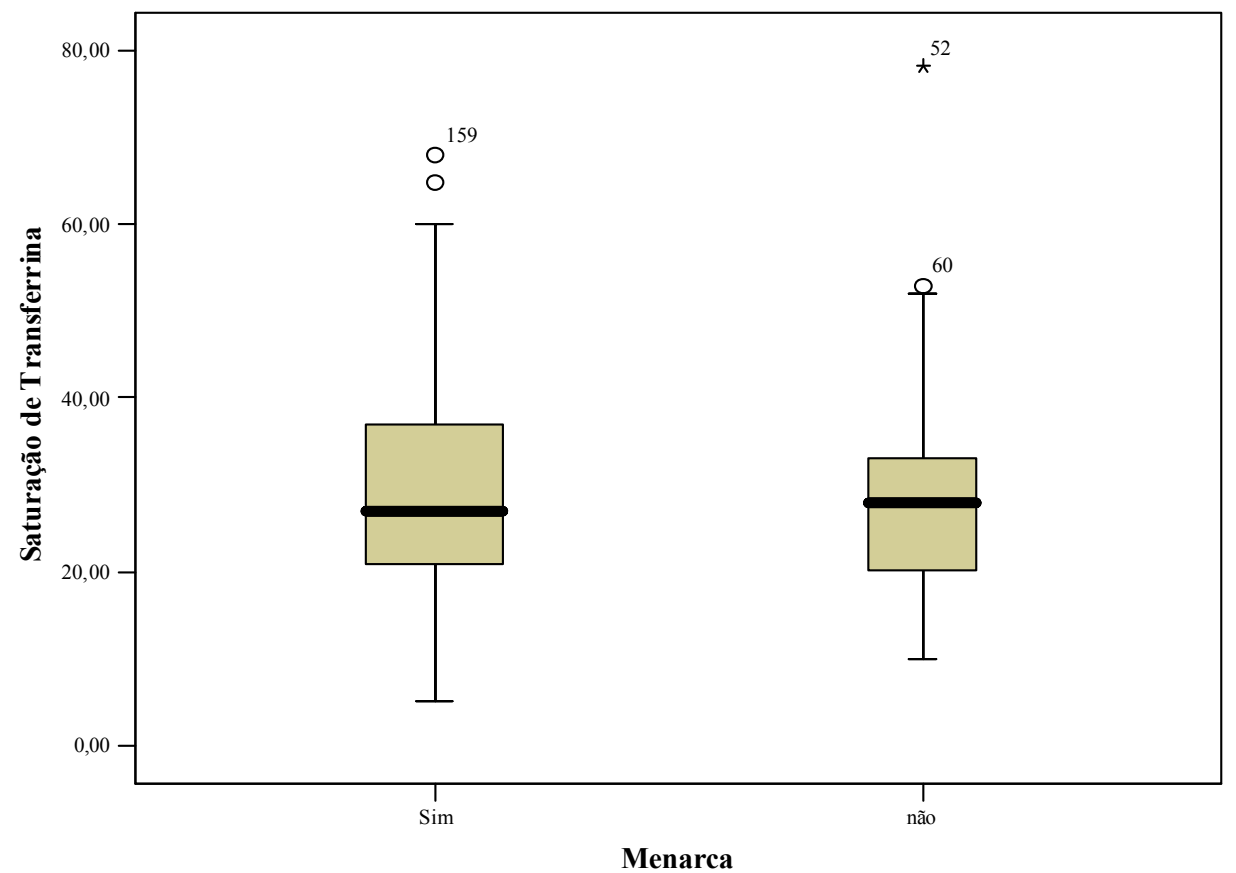

Figura 1 - Saturação de transferrina em adolescentes do sexo feminino com e sem menarca 
Por intermédio da análise de variância constatou-se que o percentual médio de saturação de transferrina em adolescentes com menarca foi significativamente diferente entre $\left(F_{(2 ; 62)}=9,72\right.$; $p=0,001)$ nas diferentes faixa etárias. Este percentual médio na faixa etária de 10 a 12 anos e 11 meses foi de 21,37 ( $d p=6,2$ ), significativamente menor pelo teste de Tukey do apresentado pelas adolescentes das outras duas faixas etárias: na faixa de 13 a 15 anos e 11 meses 36,17 ( $d p=16,74)$ e na faixa de 16 a 19 anos e 11 meses 34,18 $(\mathrm{dp}=9,48)$.

Ainda que o percentual médio de saturação de transferrina na primeira faixa tenha sido estatisticamente inferior ao observado nas outras duas, no diagnóstico de deficiência de ferro os dados não apresentaram conclusão significativa ( $p=0,392)$, ou seja, não houve associação entre deficiência de ferro e faixa etária das adolescentes com menarca.

Os dados não apresentaram associação significativa entre tipo de moradia (alvenaria e madeira) e deficiência de ferro nos adolescentes $\left(\chi_{(1)}^{2}=0,179 ; p=0,672\right)$, nem com saneamento básico (possui, não possui) $\left(\chi_{(1)}^{2}=1,307\right.$; $\mathrm{p}=0,253)$, e ou renda familiar (abaixo de $\mathrm{R} \$ 1.000,00$; acima de $\mathrm{R} \$ 1.000,00)\left(\chi_{(1)}^{2}=3,713\right.$; $p=0,054)$.

\section{DISCUSSÃO}

Na puberdade, período em que ocorre a maturação sexual, impondo-se um aumento da necessidade de ferro, ocorre um ponto máximo de crescimento com ritmos aproximados de 8 a $12 \mathrm{~cm}$ por ano para o sexo feminino e masculino respectivamente. Esse período de crescimento é denominado de "estirão pubertário". Nesse período o adolescente cresce aproximadamente $20 \mathrm{~cm}$, podendo haver uma flutuação de 10 a $30 \mathrm{~cm}$.

A diferença entre ambos os sexos se registra pela quadruplicação da massa muscular no sexo masculino e a duplicação no sexo feminino, segundo Maddaleno ${ }^{1}$. Ocorre ainda uma produção maior no nível de hemoglobina e aumento de glóbulos vermelhos provocados por estímulo da medula óssea e ativação da anidrase carbônica, diretamente exercido pela testosterona. Para todos esses acontecimentos estima - se que ao redor de $350 \mathrm{mg} /$ Fe/ano sejam necessários.

A perda sanguínea após a menarca também pode concorrer para o aparecimento da carência de ferro, podendo ser potencializada com ciclos menstruais irregulares, muito comum nos primeiros dois anos após a menarca, apesar de uma oferta nutricional normal. A perda sanguínea é considerada a segunda maior causa de deficiência de ferro, segundo FuJimori et al. ${ }^{2} \mathrm{Na}$ adolescência a dieta deve ser bem balanceada levando-se em consideração os fatores referidos anteriormente mais as perdas basais que representam aproximadamente $14 \mathrm{gg} / \mathrm{Kg} / \mathrm{dia}^{3}$.
A nutrição deficiente durante a adolescência pode ser causada por uma variabilidade de fatores, incluindo-se a instabilidade emocional, desejo obsessivo de emagrecer, instabilidade geral no estilo de vida e circunstancias sociais.

Dados do NHANES III mostram que na dieta americana, aproximadamente $27 \%$ do total energético diário são provenientes de alimentos com alto teor energético e pobres em nutrientes (junk foods) 4 .

Tem-se observado um aumento de densidade energética das dietas no Brasil, evidenciadas pela comparação de pesquisas de consumo alimentar conduzidas no país em 1961-62 (Fundação Getúlio Vargas 1970) ${ }^{5}$, em 1987-88 (IBGE - Instituto Brasileiro de Geografia e Estatística) ${ }^{6}$ e em 2008-09 (IBGE - Instituto Brasileiro de Geografia e Estatística/POFPesquisa de Orçamento Familiar, 2011)7. Está última pesquisa mostra que entre os adolescentes existe um maior consumo de açucares e colesterol. As carnes e vitamina $\mathrm{C}$ que promovem aumento da absorção do ferro não-heme são mostrados nas pesquisas como de consumo precário. Isso se deve ao preço dos alimentos que estão cada vez mais elevados, e a maioria dos brasileiros não ter renda suficiente para poder adquirir.

Constatou-se que a deficiência de ferro não foi privilégio de nenhum dos grupos distribuídos segundo o estirão pubertário. Ela esteve presente em qualquer estágio da adolescência, com prevalência considerada alta $(14,5 \%)$ quando comparada a resultados encontrados em outros estudos como de Bruneira e Castro ${ }^{8}$ que indicam prevalência ao redor de $10 \%$ para os adolescentes do sexo masculino e $5 \%$ para as do sexo feminino. Mas, em relação ao gênero, ainda que as diferenças não tenham sido estatisticamente significantes, verificou-se uma forte evidência de que a deficiência de ferro É mais prevalente entre os rapazes acentuando-se naqueles no estirão, resultado similar ao descrito por Silva ${ }^{9}$. Da mesma forma os dados obtidos no estudo realizado por Silva et al. ${ }^{10}$ durante a investigação da proporção de anemia por deficiência de ferro no estadiamento puberal, mostram maior frequência da deficiência nutricional entre os homens ( $50 \%)$ no estágio genital e nas mulheres $(30 \%)$ durante o estágio de mama. Também dados da pesquisa realizada por Iuliano et al. ${ }^{11}$, com escolares adolescentes do estado de São Paulo, sobre a prevalência de anemia por deficiência de ferro, levando em consideração a maturação sexual, detectaram grau leve de anemia em $11 \%$ dos sujeitos pesquisados, resultado que levou os autores a concluir que existe um aumento dos níveis de hemoglobina com o desenvolvimento sexual dos adolescentes.

Contrariando os achados anteriores, a pesquisa realizada por Garanito et al. ${ }^{12}$ mostra que em alguns países como a Suíça, Índia e Indonésia, as estatísticas sobre deficiência de ferro mostram ser ela é mais prevalente entre adolescentes do sexo feminino. . No mesmo sentido foram os achados 
estatísticos do levantamento realizado por Vitalle e Fisberg ${ }^{13}$ que mostram que no Paquistão, Irlanda, Reino Unido e México maior prevalência de deficiência de ferro ocorre entre adolescentes do sexo feminino. No Brasil, várias pesquisas regionais destacam as meninas adolescentes com maior índices de deficiência de ferro. Vitalle e Fisberg ${ }^{20}$ igualmente destacam que a prevalência de deficiência de ferro entre gênero feminino tem relação com a irregularidade menstrual que acontece no período após a menarca, muitas vezes tendo sangramentos abundantes ${ }^{13}$. De forma diferente, em alguns países da Europa como Espanha, Suécia e Inglaterra as estatísticas não mostraram o gênero dos adolescentes anêmicos.

Entre as pesquisas regionais no Brasil, destaca-se o estudo realizado por Mariath et al. ${ }^{14}$, com crianças e adolescentes atendidos pela equipe estratégica de saúde da família em Itajaí Santa Catariana, identificaram que as diferenças estatísticas não foram significativas entre os gêneros na avaliação de concentração hemoglobina, o hematócrito e o ferro sérico entre as crianças e adolescentes estudados.

Em Londrina, no Paraná, Miglioranza et al. ${ }^{15}$ estudaram a prevalência de anemia em escolas adolescentes da periferia daquela cidade, os resultados mostraram prevalência de $41,3 \%$ de deficiência de ferro, onde a diferença entre os sexos não foi significativa, também não ficou comprovada a relação entre deficiência de ferro e desnutrição.

Uma pesquisa realizada em Salvador, na Bahia, com adolescentes de escolas públicas, os exames de dosagem de hemoglobina de $24,5 \%$ dos participantes mostrou deficiência de ferro entre mulheres, sendo que os níveis de hemoglobina mínima foram de $7,7 \mathrm{~g} / \mathrm{dL}^{16}$.

Mesmo não existindo no Brasil, um estudo multicêntrico e nacional sobre a deficiência de ferro entre adolescentes, os pesquisadores comungam do consenso de que é alta a prevalência de deficiência de ferro entre eles, com uma estimativa de $20 \%$ de deficiência de ferro sem uma classificação de sexo já que não são dados oficiais ${ }^{13}$.

\section{REFERÊNCIAS}

1. Organização Mundial de Saúde. Status físico: o uso e a interpretação da antropometria. Genebra, 1995. (Relatório de Série Técnica, N. 854).

2. Mussen $\mathrm{PH}$, Conger JJ, Kagan J, Huston AC. Desenvolvimento e personalidade da criança. 3.ed. São Paulo: Harbra: 2001.

3. Frutuoso MFP, Vigantzky VA, Gambardella AMD. Níveis séricos de hemoglobina em adolescentes segundo estágio de maturação sexual. R. Nutr 2003;16 (2):4-11.

4. Fagioli D, Nasser LA. Educação nutricional na infância e na adolescência: planejamento, intervenção, avaliação e dinâmicas. São Paulo: RCN: 2008.
Os estudos que mostram maior prevalência entre mulheres têm como base fatores associados à menarca, maturação sexual e o estirão. Nesse sentido Azevedo et al. ${ }^{17}$ destacam que na adolescência o organismo feminino sofre mudanças rápidas tanto no crescimento como na maturação sexual, com a menarca ocorre perca natural de minerais, sendo que a eliminação de ferro do organismo acarreta a anemia ferropriva.

Os sinais e sintomas foram pouco significativos neste trabalho, o que deixa margem para afirmar que a deficiência de ferro é oligosintomática ou assintomática, sendo então de difícil diagnóstico clínico, apesar de ser considerada uma patologia que afeta a saúde dos adolescentes, e que prevalece como problema de saúde pública. é nesse sentido que Vitolo ${ }^{18}$ e Nead et al. ${ }^{19}$ confirmam que a deficiência de ferro, por si só, é um problema de saúde, mesmo que não haja quadro clínico de anemia.

A anemia é uma manifestação tardia e insidiosa da carência de ferro, em virtude do balanço negativo do mineral ${ }^{20}$. Vitalle e Fisberg ${ }^{20}$ explicam que na adolescência os sintomas da anemia são sutis, passando muitas vezes despercebidos, porém alguns sinais são marcantes como a fadiga, fraqueza e emagrecimento. Nesse mesmo sentido Almeida ${ }^{21}$ especifica que, embora de forma quase imperceptível, a deficiência de ferro apresenta quadro clínico de "palidez cutâneo-mucosa, astenia, fadiga, distúrbio alimentar e redução do crescimento", sendo que o principal comprometimento está na diminuição do transporte de oxigênio para os tecidos devido a diminuição da concentração de hemoglobina.

Dessa forma salienta-se a importância da triagem laboratorial em adolescentes para diagnóstico da deficiência de ferro, mesmo quando estes não apresentem sintomas, pois outras anormalidades bioquímicas podem acontecer, já que o ferro participa de reações enzimáticas do organismo e, certamente terão repercussões negativas tanto no no desenvolvimento físico como no desenvolvimento cognitivo diminuindo as oportunidades de integração social do individuo ferro deficiente.

5. Lima ACVM, Lima MC, Guerra MQF, Romani SAM, Eickamnn SH, Lira, PIC. Impacto do tratamento semanal com sulfato ferroso sobre o nível de hemoglobina, moribade e estado nutricional de lactantes anêmicos. Jornal de Pediatria. 2006: 82 (6): 452-57.

6. Batista Filho M, Rissin A. A transição nutricional no Brasil: tendências regionais e temporais. Caderno de Saúde Públlica 2003; 19(1): 181191.

7. Coates V, Beznos GW, Françoso LA. Medicina do Adolescente. Ed.Sarvier $2^{a}$ edição. São Paulo: 2003, p.241-245.

8. Maddaleno M. La salud del adolescente y del joven. Washington: Organización Panamericana de la Salud: 1995, p.87-93. 
9. Fujimori E, Szarfarc SC, Oliveira IMV. Prevalência de anemia e deficiência de ferro em adolescentes do sexo feminino - Taboão da Serra, SP, Brasil. Rev. latino-am. enfermagem, Ribeirão Preto, 1996; 4(3): 49-63.

10. Mahan KL, Escott-Stump S. Nutrição na adolescência. In: Mahan KL, Escott-Stump S. Krause - Alimentos, Nutrição e Dietoterapia. 11.ed. São Paulo: Roca; 1998. p.279-83.

11. Kant AK. Consumption of energy-dense, nutrient-poor foods by adult Americans: nutritional and health implications. The Third National Health and Nutrition Examination Survey, 1988-1994. Am J Clin Nut, 2000;72 (2): 929-36.

12. Fundação Getúlio Vargas. Consumo de alimentos no Brasil: pesquisa de orçamento familiar (1961-62). Rio de Janeiro: FGV, 1970.

13. IBGE - Instituto Brasileiro de Geografia e Estatística. POF Pesquisa de Orçamento Familiar (1987-88). Tabelas de Composição Nutricional dos Alimentos Consumidos no Brasil. Rio de Janeiro: IBGE, 1991, p.228-239.

14. IBGE - POF - Pesquisa de Orçamentos Familiares (2008-09). Tabelas de Composição Nutricional dos Alimentos Consumidos no Brasil. Disponível em:http://www.ibge.gov.br/home/ estatistica/populacao/condicaodevida/pof/ 2008_2009_composicao nutricional/ default.shtm. Acessado em 08 de julho de 2011.

15. Bruneira $P$, Castro HC. Anemia ferropriva. In: Coates V, Beznos GW, Françoso LA. Medicina do Adolescente. Ed.Sarvier 2 ed. São Paulo: 2003, p. 241-245.

16. Silva MC. Anemia por deficiência de ferro na adolescência. Núcleo de Estudos da Saúde da Adolescência - UERJ 2007; 4(1):19-22.

11. Silva FC, Vitalle MSS, Quaglia EC, Braga JAP, Medeiros EHGR. Proporção de anemia de acordo com o estadiamento puberal, segundo dois critérios diagnósticos. Revista de Nutrição 2007; 20(3): 297-306.
17. Iuliano BA, Frutuoso MFP, Gambardella AMD. Anemia em adolescentes segundo maturação sexual. Revista de Nutrição 2004; 17 (1): 37-43.

18. Garanito MP, Pitta TS, Carneiro JDA. Deficiência de ferro na adolescência. Revista Brasileira de Hematologia e Hemoterapia 2010; 32(2): 45-48.

19. Vitalle MSS, Fisberg M. Deficiência de ferro entre adolescentes. II Jornada de Anemia Carencial e Segurança Alimentar no Brasil. Jornadas Científicas do NISAN 2007/2008: 161-73.

20. Mariath AB, Giachini RM, Lauda LG, Grillo LP. Estado de ferro e retinol sérico entre crianças e adolescentes atendidos por equipe da Estratégia de Saúde da Família de Itajaí, Santa Catarina Ciência e Saúde Coletiva 2010; 15 ( 2): 509-516.

21. Miglioranza LHS, Matsuo T, Caballero-Córdoba GM, Dichi JB, Cyrino ES, Oliveira IBN, Martins MS, Polezer N, Dichi I. Anemia prevalence in children and adolescents from educational centers in the outskirts of Londrina, PR, Brazil. Revista de Nutrição 2002;15(2): 149-53.

22. Borges CQ, Silva RCR, Assis AMO, Pinto EJ, Fiaccone RL, Pinheiro SMC. Fatores associados à anemia em crianças e adolescentes de escolas públicas de Salvador, Bahia, Brasil. Caderno de Saúde Pública 2009; 25 (4):877-88.

23. Azevedo L, Martino HSD, Carvalho FG, Rezende, ML. Estimativa da ingestão de ferro e vitamina C em adolescentes no ciclo menstrual. Revista Ciência e Saúde Coletiva 2010; 15(1): 1359-367.

24. Vitolo MR. Nutrição: da gestação à adolescência. Rio de janeiro: Reichemann \& Affonso Editores: 2003, p.193-6.

25. Nead KG, Halferman JS, Kaczorowski JM, Auinger $P$, Weitzman M. Overweight children and adolescents: a risk group for iron deficiency. Pediatrics 2004; 134(1):104-08.

26. Beard JL. Iron requirements in adolescent females. American Socity for Nuttitional Sciences 2000; 440S-43S.

27. Almeida E. Anemia ferropriva: como diagnosticar? Lincx 201. Disponível em http:// www.lableme.com.br. Acessado em 08 de julho de 2011. 